\title{
Measurements and Simulations of Interface Behavior in Metal Thin Film Peeling Along Ceramic Substrate
}

\author{
Yueguang Wei*, Haifeng Zhao and Siqi Shu \\ LNM, Institute of Mechanics, Chinese Academy of Sciences, Beijing 100080, China; \\ *E-mail: ywei@Inm.imech.ac.cn
}

\begin{abstract}
Peeling experiments for aluminum thin film along the $\mathrm{Al}_{2} \mathrm{O}_{3}$ substrate are carried out, and the variations of external driving force (energy release rate) at the steady-state delamination of the thin film in the metal film/ceramic substrate system are measured. Additionally, theoretical modeling for the thin film delamination is also performed. Based on the bending model, three double-parameter criteria are used. Three double-parameter criteria include: (1) the interfacial fracture toughness and the separation strength, (2) the interfacial fracture toughness and the interfacial crack tip slope angle of thin film, and (3) the interfacial fracture toughness and the critical von Mises effective strain of thin film at crack tip. Based on the three double-parameter criteria, the thin film nonlinear peeling problems are solved analytically for each case. The results show that the solutions of thin film nonlinear peeling based on the bending model are very sensitive to the model parameter selections. Through analyses and comparisons to different solutions, a connection between solutions based on the bending models and based on the two-dimensional elastic-plastic finite element analysis is obtained. The effective regions of each model can be specified through comparing the present experimental result with model solutions.
\end{abstract}

Key words: metal thin film, delamination, interfacial toughness, peel test, double-parameter criterion.

\section{Introduction}

Most advanced materials are inseparable with thin films which with its particular characteristic, has been widely applying to the surface and interfacial engineering areas. The material behaviors of the thin film/substrate systems are mainly dominated by the interfacial adhesion property (strength). In order to evaluate the adhesion behaviors, a simple test method, peel test, was designed fifty years ago [1]. Due to the good advantages of the test method, such as simply operating, the test has been widely applying to many research regions [2-4]. Specifically, when both the thin film and substrate are elastic materials, the interfacial adhesion toughness can be obtained directly through measuring peeling force in peel experiment. However, 
when thin film or substrate is a ductile material, the measured peeling force is often much larger than the interfacial adhesion toughness. The phenomenon is come from the plastic dissipation due to material plastic loading and unloading deformation. In order to model the peeling force (or energy release rate) increase due to plastic dissipation, Kim and his collaborators $[5,6]$ presented a bending model to predict the plastic dissipation. Within the following decade after bending model was presented, most analyses related to the ductile thin film peeling adopted the bending model of Kim et al., e.g., [7, 8]. However, Wei and Hutchinson [9] adopted a different method from that of Kim et al. in analyzing the elastic-plastic thin film peeling problems. In Wei and Hutchinson's analysis, the thin film delamination process was simulated by using the two-dimensional elastic-plastic finite element method (FEM), except the detached part of thin film, which was described still by bending model in order to avoid the difficulty in the two-dimensional large deformation analysis. They obtained a kind of different results from that of bending model, qualitatively and quantitatively. Recently, Wei [10] adopted three different double-parameter criteria based on the bending model to obtain the different relationships between the peel force and the thin film thickness. In the present research, in order to further explore the connection of bending model solution with elastic-plastic FEM solution, and in order to assess the effectiveness for every models, a series of the peel experiments for $\mathrm{Al}$ thin film delaminated along the ceramic $\left(\mathrm{Al}_{2} \mathrm{O}_{3}\right)$ substrate are carried out. The relationship between the peel force and the thin film thickness at the steady-state delamination is measured. By comparing the experimental curves with the modeling solutions, a primary connection of both the bending model and the two-dimensional FEM model is presented.

\section{Model Descriptions}

Peeling experiments for $\mathrm{Al}$ thin film along the ceramic $\left(\mathrm{Al}_{2} \mathrm{O}_{3}\right)$ substrate are carried out for a series of thin film thickness, $t=20,50,80,100,200$, and $225 \mathrm{mi}-$ crons. There exists an adhesion layer between film and substrate with a thickness of 20 microns. The interface layer material is Epoxy with two different percentages of curing agent (Polyimide), respectively. A brittle adhesive layer is formed for a compound of Epoxy with Polyimide, 1:1, and a ductile adhesive layer is for the proportion 1:1.5. The adhesive layer thickness is 20 micron in the present experiment. Figures $1 \mathrm{a}$ and $1 \mathrm{~b}$ show the peel force varying with the peeling displacements for thin film thicknesses $t=20$ and 50 microns, respectively. Three curves in Figure 1a or in Figure 1b correspond to three samples. The adhesive layer corresponds to ductile one. From Figures $1 \mathrm{a}$ and $1 \mathrm{~b}$, the feature of the peel force variations can be described as follows: firstly, peel force varies linearly with peeling displacement, secondly a steady-state peeling process is quickly arrived when peel force attains a critical value. Figure 1c shows the experimental result of the steady-state peel force (or called energy release rate) varying with several thin film thickness. Two curves correspond to ductile and brittle interface adhesives, respectively. 


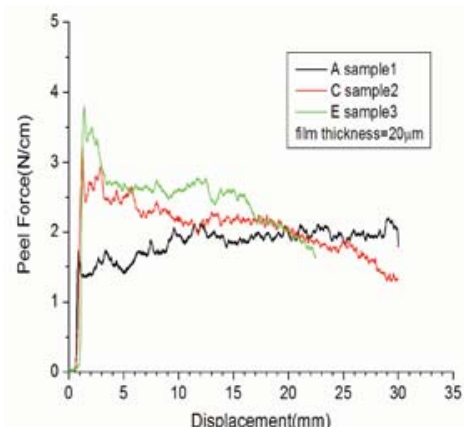

(a)

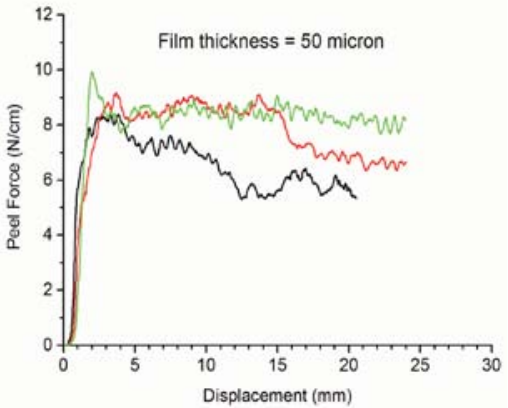

(b)

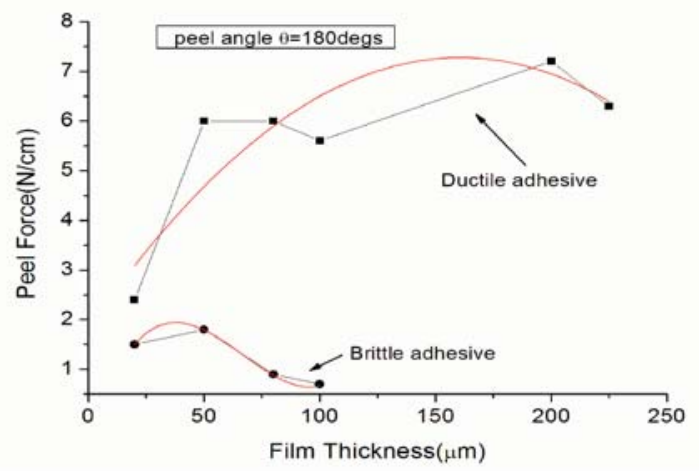

(c)

Fig. 1. Peeling experimental results.

\section{Bending Models and Delamination Criteria in Peel Test}

Delamination process of elastic-plastic thin film in peel test can be described by Figure $2 \mathrm{a}$. The thin film undergoes the delamination and plastically loading and unloading process under the act of the peel force $P$. The cross-section of the thin film is from a free-stressing state to the loading and unloading processes, as described by OABCDEF, in Figure 2a.

The process of the ductile thin film peeled and delaminated along substrate interface can be characterized by the double-parameter criterion (for elastic delamination case, single-parameter criterion is valid). Two independent parameters are needed to characterize the main characters here, the interfacial adhesion property and the plastic dissipation of the system. In the present research, three double-parameter criteria [10] will be used respectively for describing the elastic-plastic peeling process, which are given in Figures 2b, 2c and 2d, respectively. 


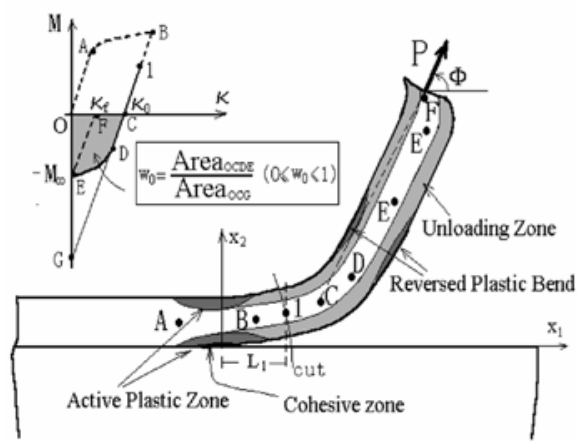

(a). Peel test

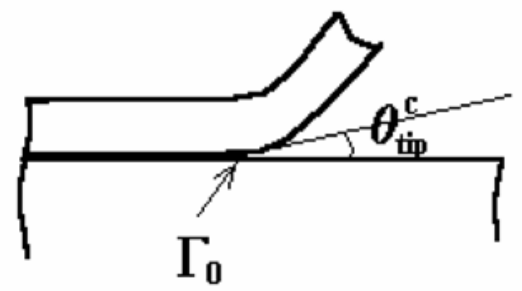

(c). $\left(\Gamma_{0}, \theta_{\text {tip }}^{c}\right)$ criterion

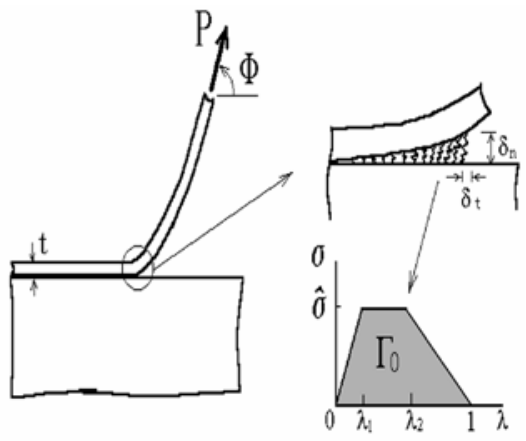

(b). $\left(\Gamma_{0}, \hat{\sigma}\right)$ criterion

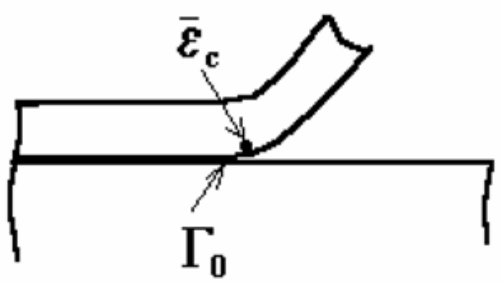

(d). $\left(\Gamma_{0}, \bar{\varepsilon}_{c}\right)$ criterion

Fig. 2. Peel test sketch (a) and three simplified double-parameter models (b)-(d) [10].

For the thin film peeling process, the relation among the peeling force $P$ per unit width of thin film (or energy release rate of system), the interfacial adhesion toughness $\Gamma_{0}$, as well as the geometrical and physical parameters of thin film and substrate is usually concerned. Under steady-state delamination, the relation can be written as:

$$
\begin{array}{llrl}
P(1-\cos \Phi) & =\Gamma_{0} & & (\text { elastic peeling) } \\
P(1-\cos \Phi) & =\Gamma_{0}+\Gamma^{p} & & \text { (elastic-plastic peeling) }
\end{array}
$$

where $\Gamma^{p}$ is the plastic dissipation. Based on the stress-strain analysis for thin film, one can obtain the fundamental relations of the thin film undergoing the nonlinear bending, furthermore, one can also obtain the plastic dissipation relation for $\Gamma^{p}$, as given in next section. 


\section{Fundamental Relations}

Kim and Aravas [5] derived out the fundamental relations based on the bending model for elastic-plastic thin film bending under the incompressible conditions $(v=1 / 2)$. The rigorous derivation based on the general case of the compressible elastic-plastic conditions is given by $[10,11]$. The relations of moment and curvature respectively for elastic, plastic and unloading cases can be dictated as follows:

$$
\begin{aligned}
\frac{M}{M_{0}} & =\frac{2 \kappa}{3 \kappa_{e}} ; \frac{M}{M_{0}}=\left\{\frac{2}{3}-\frac{2}{N+2} \gamma\right\} \frac{1}{\left(\kappa / \kappa_{e}\right)^{2}}+\frac{2}{N+2} \gamma\left(\frac{\kappa}{\kappa_{e}}\right)^{N} ; \\
\frac{M}{M_{0}} & =\frac{2}{3} \frac{\kappa-\kappa_{0}}{\kappa_{e}},
\end{aligned}
$$

and curvature relation:

$$
\kappa=\sqrt{[1-\cos (\phi-\theta)] \frac{2 P}{B}+\left(1-w_{0}\right) \kappa_{0}^{2}}, \quad \theta_{B} \approx \theta_{\text {tip }} \leq \theta \leq \theta_{C} .
$$

where $M_{0}=3 / 2 M_{e}$ is the limit bending moment for elastic-perfectly plastic material; $M_{e}$ and $\kappa_{e}$ are the elastic limit moment and elastic limit curvature, respectively,

$$
\begin{aligned}
& M_{e}=\frac{2}{3} M_{0}=\frac{\sigma_{Y} t^{2}}{6 \sqrt{1-v+v^{2}}}, \quad \kappa_{e}=\frac{2\left(1-v^{2}\right) \sigma_{\gamma}}{E t \sqrt{1-v+v^{2}}} \\
& \gamma=2{\sqrt{\frac{1}{3}\left(1-v+v^{2}\right)}}^{1-N} \quad(1-v)^{N} .
\end{aligned}
$$

$B=E t^{3} / 12\left(1-v^{2}\right)$ is the bending modulus; $w_{0}\left(0 \leq w_{0} \leq 1\right)$ is defined in Figure 1a which characterizes the inversely plastic behavior (or Bauschinger effect); $\theta_{\text {tip }}$ is the crack tip slope angle at thin film delamination; $N$ is material strain hardening exponent. For incompressible material $v=0.5$ and $\gamma=1$ expression (2) comes to the result of Kim and Aravas [5].

Suppose that substrate is rigid or Young's modulus of substrate is much larger than that of thin film, by means of formulae (2) $(M-\kappa$ relations in sketch of Figure $2 \mathrm{a}$ ), one can obtain the plastic dissipation relation through calculating the area within the circuit OABCDEO under $M-\kappa$ curve,

$$
\begin{aligned}
\Gamma^{P}= & \frac{1}{2} M_{e} \kappa_{e}-\frac{1}{2} M_{B}\left(\kappa_{B}-\kappa_{0}\right)+\left(\frac{2}{3}-\frac{2}{N+2} \gamma\right) M_{0}\left(\kappa_{e}-\frac{\kappa_{e}^{2}}{\kappa_{B}}\right) \\
& +\frac{2 \gamma}{(N+1)(N+2)} M_{0}\left(\frac{\kappa_{B}^{N+1}}{\kappa_{e}^{N}}-\kappa_{e}\right)+\frac{1}{2} B \kappa_{0}^{2} w_{0}
\end{aligned}
$$

\section{Bending Model Solutions of Thin Film Nonlinear Peeling}

Based on three double-parameter criteria, the peel force can be solved for peeling process. The solving procedures can be dictated as follows. Firstly, from (3) a relation 


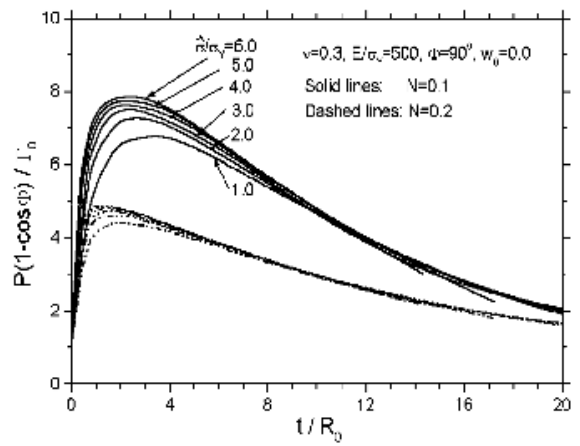

(a)

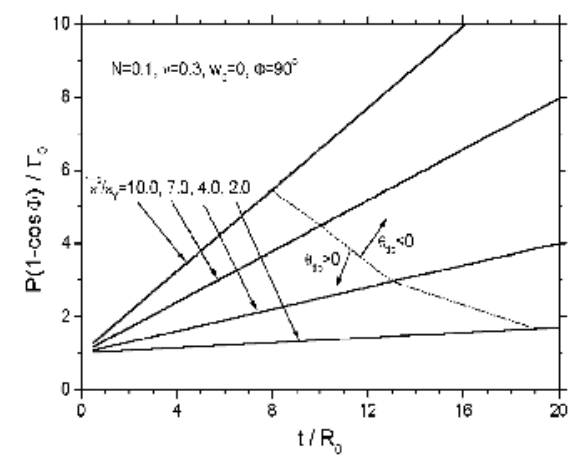

(c)

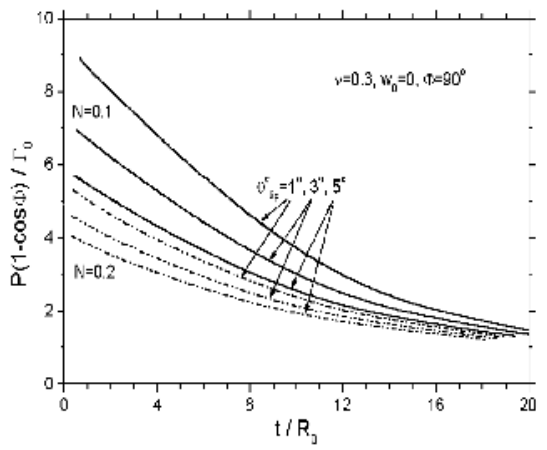

(b)

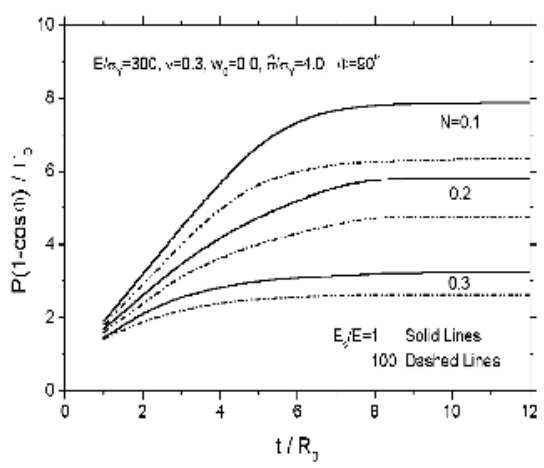

(d)

Fig. 3. Results based on three double-parameter criteria (a)-(c) and two-dimensional finite element method (d).

among the parameters $\left(\kappa_{B}, P, \theta_{\text {tip }}=\theta_{B}, \kappa_{0}\right)$ can be obtained to each criterion. Then the parameters $\left(M_{B}, \kappa_{B}, \kappa_{0}\right)$ can be solved simultaneously with Equations (2) for $M=M_{B}$ and $\kappa=\kappa_{B}$. Finally, the peel force variations with related parameters can be attained from (1) and (5).

The solution forms by adopting three double-parameter criteria can be given through independent parameters by dimensional analysis. They are dictated as

$$
\begin{gathered}
\left\{\begin{array}{l}
\frac{P(1-\cos \Phi)}{\Gamma_{0}}=f_{1}\left(E / \sigma_{Y}, \hat{\sigma} / \sigma_{Y}, N, v, t / R_{0}, w_{0}, \Phi\right) \quad\left(\text { for }\left(\Gamma_{0}, \hat{\sigma}\right) \text { criterion }\right) ; \\
\theta_{\text {tip }}=r_{1}\left(E / \sigma_{Y}, \hat{\sigma} / \sigma_{Y}, N, v, t / R_{0}, w_{0}, \Phi\right)
\end{array}\right. \\
\frac{P(1-\cos \Phi)}{\Gamma_{0}}=f_{2}\left(N, v, t / R_{0}, w_{0}, \Phi, \theta_{\text {tip }}^{c}\right) \quad\left(\text { for }\left(\Gamma_{0}, \theta_{\text {tip }}^{c}\right) \text { criterion }\right) ;
\end{gathered}
$$




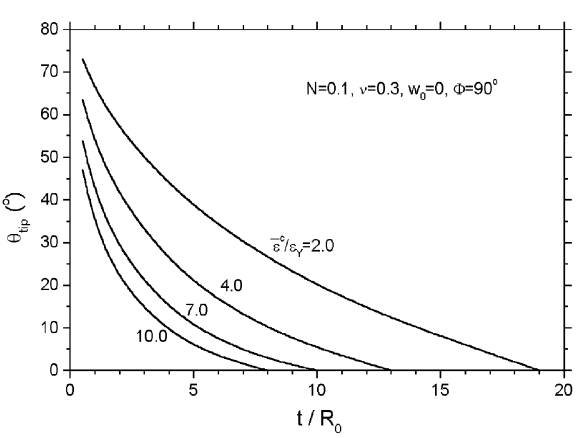

(a)

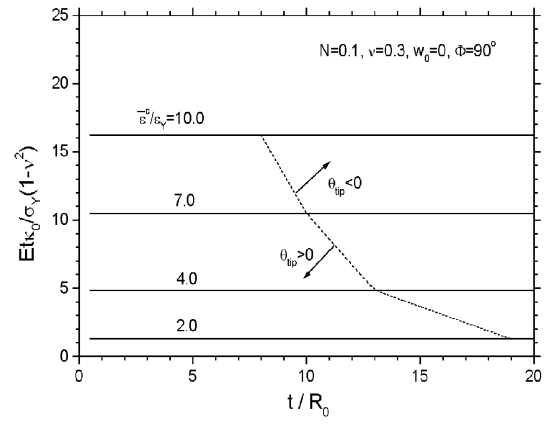

(b)

Fig. 4. Variations of the thin film inclined angle at tip and the residual curvature with thin film thickness based on the criterion $\left(\Gamma_{0}, \bar{\varepsilon}_{c}\right)$.

$$
\left\{\begin{array}{l}
\frac{P(1-\cos \Phi)}{\Gamma_{0}}=f_{3}\left(N, v, t / R_{0}, w_{0}, \Phi, \bar{\varepsilon}_{c} / \varepsilon_{Y}\right) \\
\theta_{\text {tip }}=r_{3}\left(N, v, t / R_{0}, w_{0}, \Phi, \bar{\varepsilon}_{c} / \varepsilon_{Y}\right)
\end{array} \quad \text { (for }\left(\Gamma_{0}, \bar{\varepsilon}_{c}\right)\right. \text { criterion) }
$$

where a length parameter $R_{0}$ is introduced and is defined as $R_{0}=E T_{0} / 3 \pi(1-$ $\left.v^{2}\right) \sigma_{Y}^{2}$, which characterizes the plastic zone size at crack tip under the small scale yielding case. $\theta_{\text {tip }}$ is crack tip slope angle. $\varepsilon_{Y}=\sigma_{Y} / E$ is yielding strain.

The solutions based on three double-parameter criteria $\left(\Gamma_{0}, \hat{\sigma}\right),\left(\Gamma_{0}, \theta_{\text {tip }}^{c}\right)$ and $\left(\Gamma_{0}, \bar{\varepsilon}_{c}\right)$ are given in Figures 3a-3c, respectively. Results in Figure 3 show the curves of the normalized energy release rate (or peeling force) versus normalized thin film thickness under elastic-plastic steady-state delamination. If thin film deformation is elastic, $P(1-\cos \Phi) / \Gamma_{0}=1$, see first relation of formula (1). Clearly, the energy release rate is remarkably enlarged by the plastic dissipation. From Figure 3a, the variation of the normalized peeling force (energy release rate) $P(1-\cos \Phi) / \Gamma_{0}$ with thin film thickness can be described as follows. Its value tends to 1 when thin film thickness is very small, i.e., the effect of plastic dissipation can be neglected. As thin film thickness increases, energy release rate increases sharply and obtains a maximum value at about $t / R_{0}=2$. The plastic dissipation decreases gradually as thin film thickness increases further. The results shown in Figure $3 \mathrm{~b}$ are based on the $\left(\Gamma_{0}, \theta_{\text {tip }}^{c}\right)$ criterion. From the results, the variation of $P(1-\cos \Phi) / \Gamma_{0}$ versis $t / R_{0}$ is much different from that based on the double-parameter criterion $\left(\Gamma_{0}, \hat{\sigma}\right)$. Here, the value of $P(1-\cos \Phi) / \Gamma_{0}$ increases always as thin film thickness decreases. This trend is consistent with that given by Kim and his collaborators for incompressible material $[5,6]$. The results based on the $\left(\Gamma_{0}, \bar{\varepsilon}_{c}\right)$ criterion are given in Figure $3 \mathrm{c}$. Obviously, the variation of $P(1-\cos \Phi) / \Gamma_{0}$ versus $t / R_{0}$ is different from those shown above based on the other double-parameter criteria. In Figure $3 \mathrm{c}, P(1-\cos \Phi) / \Gamma_{0}$ increases linearly with increasing $t / R_{0}$. The inclined angles of the straight line increase with increasing the critical von Mises effective strain at tip. In order to investigate the third criterion, Figures $4 \mathrm{a}$ and $4 \mathrm{~b}$ show the variations of the crack tip slope angle and the residual curvature. When thin film thickness is small, the slope angle 
is large. With increasing thin film thickness, the slope angle decreases sharply and transits to negative value from positive value. Obviously, the negative slope angle of thin film at tip violates the physical requirement. It implies that the criterion is failure and the predicted results, top part of the dashed line in Figure 3c, should be cut off. The reason can be interpreted in referring to Figure $4 \mathrm{~b}$ that when thin film thickness is small, the large critical Mises effective strain at crack tip is realized by large bending deformation (curvature) in thin film, however when thin film thickness is large, it is difficult or even impossible to realize the large bending curvature of thin film to meet with the high critical Mises effective strain, and in this case the criterion $\left(\Gamma_{0}, \bar{\varepsilon}_{c}\right)$ is failure.

In order to assess the bending model solutions based on the above three twoparameter critera, the two-dimensional elastic-plastic finite element results [9] are also shown here, see Figure 3d. From Figure 3d, the bending model results based on the third double-parameter criterion have the similar variation trends and quantity with those of the elastic-plastic finite element calculation within the region of about $t / R_{0}<5$ (in Figure 3d, $E_{s}$ is Young's modulus of elastic substrate). However, as thin film thickness increases continuously, the finite element solution tends to the small scale yielding solution (insensitive to thin film thickness), while the bending model solution based on the double-parameter criterion is failure. Therefore, when thin film thickness is large, i.e., $t / R_{0}>5$, how to set up a governing parameter criterion based on the bending model will be a tough task to need to be explored in the future.

\section{Comparison of Experiment Results with Modeling Results}

Peeling experimental results of the relationship between the energy release rate and the film thickness at the steady-state delamination are shown in Figure 1c. Modeling results based on the bend model three criteria and the two-dimensional finite element method are shown in Figure 3. Through comparing the experimental results with the modeling results, the following key points can be read:

1. Both parameter criteria, $\left(\Gamma_{0}, \hat{\sigma}\right)$ and $\left(\Gamma_{0}, \theta_{\text {tip }}^{c}\right)$, are suitable for a weaker-adhesion interface case, while two-dimensional elastic-plastic analysis (finite element simulation) is suitable for a stronger-adhesion interface case.

2. When film thickness is very thin, such as for $t / R_{0}<5$, the criterion $\left(\Gamma_{0}, \theta_{\text {tip }}^{c}\right)$ is failure, while the other criteria mentioned above are valid.

\section{Discussions to Bending Model Solutions}

In the present research, the peel experiments for $\mathrm{Al}$ thin film delaminated along the ceramic $\left(\mathrm{Al}_{2} \mathrm{O}_{3}\right)$ substrate have been carried out. The relationship between the peel force and the thin film thickness at the steady-state film delamination has been measured. By comparing the experimental curves with the modeling solutions, the effect- 
ive regions for each analysis models has been assessed, and a primary connection of both the bending model and the two-dimensional FEM model has been developed.

The bending model solutions of thin film nonlinear peeling based on the three double-parameter criteria have been obtained in last section. Obviously, the bending model solutions are very sensitive to the selection of the governing parameters. This leads to a question: what is a reasonable selection of the governing parameter group for predicting the thin film nonlinear peeling by using the bending model? From the solutions based on the first and second double-parameter criteria, when normalized thin film thickness is smaller than about $5, t / R_{0}<5$, the contribution of bending plastic dissipation to energy release rate is very large. This can be confirmed from variation of the residual curvature in [11]. However, from the solutions based on the criterion of the interfacial fracture toughness and the critical Mises effective strain, when thin film thickness is smaller than about $5, t / R_{0}<5$, the contribution of the bending plastic dissipation to energy release rate is obviously smaller than that based on the first or second criterion.

Through comparing the experimental results with the modeling results, one can conclude that both parameter criteria, $\left(\Gamma_{0}, \hat{\sigma}\right)$ and $\left(\Gamma_{0}, \theta_{\text {tip }}^{c}\right)$, are suitable for a weaker-adhesion interface case, while two-dimensional elastic-plastic analysis (finite element simulation) is suitable for a stronger-adhesion interface case, however when film thickness is very thin, such as for $t / R_{0}<5$, the criterion $\left(\Gamma_{0}, \theta_{\text {tip }}^{c}\right)$ is failure, while the other criteria mentioned above are valid.

\section{Acknowledgements}

The work is supported by National Science Foundations of China through Grants 10432050 and 10428207.

\section{References}

1. Spies, G.J., The peeling test on redux-bonded joints. J. Aircraft Eng. 25, 1953, 64.

2. Feliu-Baez, R., Lockhart, H.E. and Burgess G., Correlation of peel and burst tests for pouches. Packaging Tech. Sci. 14, 2001, 63.

3. Choi, J.W. and Oh, T.S., Peel strength and peel angle measured by the T-peel test on Cr/BPDA-PDA interface. J. Adhe. Sci. Tech. 15, 2001, 139.

4. Asai, H., Iwase, N. and Suga, T., Influence of ceramic surface treatment on peel-off strength between aluminum nitride and epoxy-modified polyaminobismaleimide adhesive. IEEE Trans. on Adv. Packaging 24, 2001, 104.

5. Kim, K.S. and Aravas, N., Elasto-plastic analysis of the peel test. Int. J. Solids Struct. 24, 1988, 417.

6. Kim, J., Kim, K.S. and Kim, Y.H., Mechanical effects of peel adhesion test. J. Adhe. Sci. Tech. 3, 1989, 175.

7. Kinloch, A.J., Lau, C.C. and Williams, J.G., The peeling of flexible laminates. Int. J. Fracture 66, 1994, 45.

8. Moidu, A.K., Sinclair, A.N. and Spelt, J.K., On determination of fracture energy using the peel test. J. Testing Evalu. 26, 1998, 247. 
9. Wei, Y. and Hutchinson, J.W., Interface strength, work of adhesion and plasticity in the peel test. Int. J. Fracture 93, 1998, 315.

10. Wei, Y., Modeling nonlinear peeling of ductile thin films - Critical assessment of analytical bending models using FE simulations. Int. J. Solid \& Structures 41, 2004, 5087.

11. Wei, Y. and Hutchinson, J.W., Peel test and interfacial toughness, in Encyclopedia of Comprehensive Structural Integrity, Vol. 8, W.W. Gerberich and W. Yang (eds.), Chapter 5, Elsevier Science Publisher, 2003, p. 181. 